\title{
A mean value density theorem of additive number theory
}

by

\author{
Friedrich RoEsler (München)
}

Let $A$ be a finite set of integers and

$$
A+A=\{a+b: a, b \in A\}, \quad A-A=\{a-b: a, b \in A\}
$$

be the sum set and the difference set of $A$. We denote by

$$
S(A)=|A+A|, \quad D(A)=|A-A|
$$

the cardinality of these sets.

There should be intrinsic connections between $A+A$ and $A-A$, for the nontrivial coincidences $a+b=a^{\prime}+b^{\prime}$ of sums are equivalent to the nontrivial coincidences $a-a^{\prime}=b^{\prime}-b$ of differences.

If $A$ has $k$ elements, then obviously

$$
2 k-1 \leq S(A) \leq\left(\begin{array}{c}
k+1 \\
2
\end{array}\right), \quad 2 k-1 \leq D(A) \leq k^{2}-k+1 .
$$

If $A=\{1, \ldots, k\}$ or more generally if $A$ is an arithmetic progression of $k$ integers, then $S(A)=D(A)=2 k-1$ and hence

$$
\frac{D(A)}{S(A)}=1 \text {. }
$$

If the $k$ elements of $A$ form a sufficiently fast growing sequence, then there are no nontrivial coincidences and thus $S(A)=\left(\begin{array}{c}k+1 \\ 2\end{array}\right), D(A)=k^{2}-k+1$, and

$$
\frac{D(A)}{S(A)}=1+\left(1-\frac{2}{k}\right)\left(1-\frac{2}{k+1}\right)<2 .
$$

Nevertheless the general conjecture

$$
1 \leq \frac{D(A)}{S(A)}<2
$$

2000 Mathematics Subject Classification: Primary 11B05. 
is false. G. A. Freĭman and V. P. Pigarev [1] have constructed arbitrarily large sets $A$ and $A^{\prime}$ such that

$$
\frac{D(A)}{S(A)}>D(A)^{0.11} \text { and } \frac{D\left(A^{\prime}\right)}{S\left(A^{\prime}\right)}<D\left(A^{\prime}\right)^{-0.017} .
$$

These sets are designed explicitly to violate (1) (comp. also [5]). But even natural born sets like $A_{k}=\left\{m^{2}: 0 \leq m<k\right\}, k=1,2, \ldots$, are far from obeying the estimate in (1): E. Landau's theorem [3, p. 643] on the number of integers $n \leq x$ which have a representation as a sum of two squares, combined with a theorem of G. Tenenbaum [2, p. 29, Theorem 21(ii)] on the number of integers $n \leq x$ having a divisor in the interval $] \sqrt{x} / 2, \sqrt{x}]$, shows

$$
\lim _{k \rightarrow \infty} \frac{D\left(A_{k}\right)}{S\left(A_{k}\right)}=\infty
$$

for the sequence $A_{\infty}=\left(m^{2}\right)_{m \geq 0}$ of squares.

Here we will prove a mean value version of (1):

TheOREM. We have

$$
1 \leq \frac{D(k, N)}{S(k, N)}<2 \quad \text { for } 1 \leq k \leq N
$$

with

$$
\begin{aligned}
S(k, N) & :=\sum_{A \subset\{0,1, \ldots, N-1\},|A|=k} S(A), \\
D(k, N) & :=\sum_{A \subset\{0,1, \ldots, N-1\},|A|=k} D(A) .
\end{aligned}
$$

Both the lower bound 1 as well as the upper bound 2 in the Theorem are best possible (Remark 3 ).

The computation of $S(k, N)$ is straightforward (Proposition 1), whereas the treatment of $D(k, N)$ (Propositions 2 and 3 ) is more delicate. The reason is as follows:

To calculate $S(k, N)$ we have to count the number of subsets $A$ with $k$ elements in $\{0,1, \ldots, N-1\}$ such that $t \in A+A$ for given values $t$, i.e.

$$
\sigma_{t}(k, N):=|\{A \subset\{0,1, \ldots, N-1\}:|A|=k, t \in A+A\}| .
$$

Hence $A$ is counted in $\sigma_{t}(k, N)$ if and only if $A$ contains one of the sets

$$
\{j, t-j\}, \quad 0 \leq j \leq t / 2 .
$$

Concerning $D(k, N)$ we look at the number of subsets $A$ such that $t \in A-A$, i.e.

$$
\delta_{t}(k, N):=|\{A \subset\{0,1, \ldots, N-1\}:|A|=k, t \in A-A\}| .
$$

$A$ is counted in $\delta_{t}(k, N)$ if and only if $A$ contains one of the sets

$$
\{j, t+j\}, \quad 0 \leq j \leq N-1-t .
$$


The sets in (2) are pairwise disjoint, and therefore $\sigma_{t}(k, N)$ is given by a simple combinatorial formula. But the sets in (3) may have nonempty intersections, and this complicates the computation of $\delta_{t}(k, N)$.

We restrain from developing an exact formula for $D(k, N)$. If $k$ is not too small, then $D_{0}(k, N)$ in Proposition 3(2) is a fairly good approximation of $D(k, N)$; it is better than indicated by the error term $2 \theta\left(\begin{array}{c}N+1 \\ k+1\end{array}\right)^{*}$ (comp. Remark 2) and precisely small enough to prove the Theorem.

The technical computations in the proofs suggest introducing the coefficients

$$
\left(\begin{array}{c}
N \\
k
\end{array}\right)^{*}:=\left(\begin{array}{l}
N \\
k
\end{array}\right)- \begin{cases}2^{k}\left(\begin{array}{c}
M \\
k
\end{array}\right) & \text { if } N=2 M \\
2^{k-1}\left(\left(\begin{array}{c}
M \\
k
\end{array}\right)+\left(\begin{array}{c}
M+1 \\
k
\end{array}\right)\right) & \text { if } N=2 M+1 .\end{cases}
$$

A combinatorial interpretation of these numbers is given in Remark 1.

Repeatedly we will have to handle the cases " $N$ even" and " $N$ odd" separately. Then we write $N=2 M+\delta, 0 \leq \delta \leq 1$.

The passage from the false estimate (1) to the mean value theorem "kills the arithmetic interest of the question" (J.-M. Deshouillers) which actual value is adopted by the quotient $D(A) / S(A)$ for a given set $A$. The estimate in the Theorem, and in some more detail the graph of the function $k \mapsto D(k, N) / S(k, N), 1 \leq k \leq N$ (comp. Remark 3), just describes an average density property of finite sets $A$. But perhaps it might serve as an intuitive clue in the examination of sets as to relative density of their sum and difference set.

If a growing sequence $A_{\infty}=\left(a_{m}\right)_{m \geq 0}$ of integers is very smooth, then, with $A_{k}=\left(a_{m}\right)_{0 \leq m<k}$, one may expect the sequence

$$
\frac{D\left(A_{k}\right)}{S\left(A_{k}\right)}, \quad k=0,1,2, \ldots,
$$

to converge. In the case of the squares $a_{m}=m^{2}$ it does, even if not to a value between 1 and 2 . Similarly, if $a_{m}=\left(\begin{array}{c}m \\ 2\end{array}\right)$, then the sequence of quotients in (4) seems to grow in principle, too. On the other hand, if $a_{m}=$ $\left[\mathrm{m}^{3 / 2}\right]$, then the quotients in (4) probably fall to the limit 1 . But what kind of arithmetic properties or lack of such properties in $A_{\infty}$ might cause the sequence $D\left(A_{k}\right) / S\left(A_{k}\right)$ to grow or to fall or to converge at all?

I am grateful to J.-M. Deshouillers for his comments regarding existing results related to this work.

We shall make use of the following combinatorial results: 
LEMMA 1. We have

(1) $\sum_{j \geq 0}(-1)^{j}\left(\begin{array}{c}M \\ j\end{array}\right)\left(\begin{array}{c}2 M-2 j \\ 2 M-k\end{array}\right)=2^{k}\left(\begin{array}{c}M \\ k\end{array}\right)$.

(2) $\sum_{j \geq 0}(-1)^{j}\left(\begin{array}{c}m \\ j\end{array}\right)\left(\begin{array}{c}N-2 j \\ k\end{array}\right)=\sum_{j \geq 0} 2^{j}\left(\begin{array}{c}m \\ j\end{array}\right)\left(\begin{array}{c}N-2 m \\ N-k-j\end{array}\right)$

$$
\text { for } 0 \leq m \leq N / 2 \text {. }
$$

Pr o of. (1) Riordan [4, p. 37, line 10]; (2) from part (1) by induction on $k$ and $N$.

Lemma 2. Let $N=2 M+\delta, 0 \leq \delta \leq 1$.

(1) $\left(\begin{array}{c}N+2 \\ k+2\end{array}\right)^{*}=\left(\begin{array}{c}N \\ k+2\end{array}\right)^{*}+2\left(\begin{array}{c}N \\ k+1\end{array}\right)^{*}+\left(\begin{array}{c}N \\ k\end{array}\right)$.

(2) $\left(\begin{array}{c}N+1 \\ k+1\end{array}\right)^{*}=\left(\begin{array}{c}N \\ k+1\end{array}\right)^{*}+\left(\begin{array}{c}N \\ k\end{array}\right)^{*}+\delta \cdot 2^{k-1}\left(\begin{array}{c}M \\ k-1\end{array}\right)$.

(3) $\left(\begin{array}{c}N+2 \\ k+2\end{array}\right)^{*}=\left(\begin{array}{c}N+1 \\ k+2\end{array}\right)^{*}+\left(\begin{array}{c}N \\ k+1\end{array}\right)^{*}+\left(\begin{array}{c}N \\ k\end{array}\right)-\delta \cdot 2^{k}\left(\begin{array}{c}M \\ k\end{array}\right)$.

(4) $\left(\begin{array}{c}N \\ k\end{array}\right)^{*}=\sum_{j \geq 0}(-1)^{j}\left(\begin{array}{c}M \\ j+1\end{array}\right)\left(\begin{array}{c}N-2-2 j \\ N-k\end{array}\right)$.

(5) $\left(\begin{array}{c}2 M \\ k\end{array}\right)^{*}=\sum_{j \geq 1} 2^{k-2 j}\left(\begin{array}{c}k-j \\ j\end{array}\right)\left(\begin{array}{c}M \\ k-j\end{array}\right)$.

(6) $4\left(\begin{array}{c}N \\ k+2\end{array}\right)^{*}+2\left(\begin{array}{c}N+1 \\ k+1\end{array}\right)^{*} \leq(2 N+3)\left(\begin{array}{c}N \\ k\end{array}\right)+2^{k+2}\left(\begin{array}{c}M \\ k+1\end{array}\right)$.

Pr o of. (1)-(3) immediate; (4) from Lemma 1(1); (5) by induction on $k$ and $M ;(6)$ from part (1) by induction on $k$ and $N$.

First we deal with the mean value $S(k, N)$ for the sum sets.

Proposition 1. (1) For $1 \leq k \leq N$ and $N=2 M+\delta, 0 \leq \delta \leq 1$,

$$
S(k, N)=(2 N+1)\left(\begin{array}{c}
N \\
k
\end{array}\right)+2^{k}\left(\begin{array}{c}
M \\
k
\end{array}\right)-2\left(\begin{array}{c}
N+1 \\
k+2
\end{array}\right)^{*}-2\left(\begin{array}{c}
N+2 \\
k+2
\end{array}\right)^{*} .
$$

(2) For $k \geq 2, S(k, N)$ satisfies the recursion

$$
\begin{aligned}
S(k, N)= & S(k, N-1)+S(k-1, N-2) \\
& +(2 N-1)\left(\begin{array}{c}
N-2 \\
k-2
\end{array}\right)+2^{k-1}\left(\begin{array}{c}
M-1 \\
k-1
\end{array}\right)-2\left(\begin{array}{c}
N-2 \\
k
\end{array}\right)^{*} .
\end{aligned}
$$


Proof. (1) By definition of $\sigma_{t}(k, N)$ we have

$$
S(k, N)=\sum_{t=0}^{2 N-2} \sigma_{t}(k, N)
$$

If $A \subset\{0,1, \ldots, N-1\}$ and $A^{\prime}=\{N-1-a: a \in A\}$, then $N-1-i \in$ $A+A$ if and only if $N-1+i \in A^{\prime}+A^{\prime}$. Hence

$$
\sigma_{N-1-i}(k, N)=\sigma_{N-1+i}(k, N), \quad 0 \leq i \leq N-1,
$$

and therefore

$$
S(k, N)=2 \sum_{t=0}^{N-1} \sigma_{t}(k, N)-\sigma_{N-1}(k, N) .
$$

Next we compute $\sigma_{t}(k, N)$. If $t=2 m-1$ is odd, then

$$
\begin{aligned}
\sigma_{2 m-1}(k, N)=\left(\begin{array}{c}
N \\
k
\end{array}\right)-\sum_{j \geq 0}(-1)^{j}\left(\begin{array}{c}
m \\
j
\end{array}\right)\left(\begin{array}{c}
N-2 j \\
N-k
\end{array}\right), \\
\quad 0 \leq 2 m-1 \leq N-1,
\end{aligned}
$$

since $A \subset\{0,1, \ldots, N-1\}$ with $|A|=k$ is counted in $\sigma_{2 m-1}(k, N)$ if and only if $A$ contains one of the $m$ pairwise disjoint sets $\{0,2 m-1\}$, $\{1,2 m-2\}, \ldots,\{m-1, m\}$.

If $t=2 m$ is even, then

$$
\sigma_{2 m}(k, N)=\left(\begin{array}{c}
N \\
k
\end{array}\right)-\sum_{j \geq 0}(-1)^{j}\left(\begin{array}{c}
m \\
j
\end{array}\right)\left(\begin{array}{c}
N-1-2 j \\
N-1-k
\end{array}\right), \quad 0 \leq 2 m \leq N-1 .
$$

For $A \subset\{0,1, \ldots, N-1\}$ with $|A|=k$ is counted in $\sigma_{2 m}(k, N)$ if and only if $A$ contains one of the pairwise disjoint sets $\{0,2 m\},\{1,2 m-1\}, \ldots$, $\{m-1, m+1\},\{m\}$.

Hence $\sigma_{2 m}(k, N)$ counts all $\left(\begin{array}{c}N-1 \\ k-1\end{array}\right)$ sets $A$ with $m \in A$ and

$$
\left(\begin{array}{c}
N-1 \\
k
\end{array}\right)-\sum_{j \geq 0}(-1)^{j}\left(\begin{array}{c}
m \\
j
\end{array}\right)\left(\begin{array}{c}
N-1-2 j \\
N-1-k
\end{array}\right)
$$

sets $A$ such that $m \notin A$.

Equations (6) and (7) and Lemma 1(1) yield in particular

$$
\sigma_{N-1}(k, N)=\left(\begin{array}{c}
N \\
k
\end{array}\right)-2^{k}\left(\begin{array}{c}
M \\
k
\end{array}\right) .
$$

Finally (5)-(8) show

$$
S(k, N)=2\left(\sum_{m=0}^{M-1+\delta} \sigma_{2 m}(k, N)+\sum_{m=1}^{M} \sigma_{2 m-1}(k, N)\right)-\sigma_{N-1}(k, N)
$$




$$
\begin{aligned}
= & 2 \sum_{m=0}^{M-1+\delta}\left(\left(\begin{array}{l}
N \\
k
\end{array}\right)-\sum_{j \geq 0}(-1)^{j}\left(\begin{array}{c}
m \\
j
\end{array}\right)\left(\begin{array}{c}
N-1-2 j \\
N-1-k
\end{array}\right)\right) \\
& +2 \sum_{m=1}^{M}\left(\left(\begin{array}{c}
N \\
k
\end{array}\right)-\sum_{j \geq 0}(-1)^{j}\left(\begin{array}{c}
m \\
j
\end{array}\right)\left(\begin{array}{c}
N-2 j \\
N-k
\end{array}\right)\right) \\
& -\left(\left(\begin{array}{c}
N \\
k
\end{array}\right)-2^{k}\left(\begin{array}{c}
M \\
k
\end{array}\right)\right) \\
= & (2 N+1)\left(\begin{array}{c}
N \\
k
\end{array}\right)+2^{k}\left(\begin{array}{c}
M \\
k
\end{array}\right) \\
& -2 \sum_{j \geq 0}(-1)^{j}\left(\begin{array}{c}
M+\delta \\
j+1
\end{array}\right)\left(\begin{array}{c}
N-1-2 j \\
N-1-k
\end{array}\right) \\
& -2 \sum_{j \geq 0}(-1)^{j}\left(\begin{array}{c}
M+1 \\
j+1
\end{array}\right)\left(\begin{array}{c}
N-2 j \\
N-k
\end{array}\right) \\
= & (2 N+1)\left(\begin{array}{c}
N \\
k
\end{array}\right)+2^{k}\left(\begin{array}{c}
M \\
k
\end{array}\right)-2\left(\begin{array}{c}
N+1 \\
k+2
\end{array}\right)^{*}-2\left(\begin{array}{c}
N+2 \\
k+2
\end{array}\right)^{*}
\end{aligned}
$$

by Lemma 2(4).

(2) Direct computation with Lemma $2(2,3)$.

Now we start to estimate the mean value $D(k, N)$ for the difference sets.

Proposition 2. (1) For $2 \leq k \leq N$ and $1 \leq t \leq N-1$,

$$
\delta_{t}(k, N)=\delta_{t}(k, N-1)+\delta_{t}(k-1, N-2)+\left(\begin{array}{c}
N-2 \\
k-2
\end{array}\right)+E_{t}(k, N)
$$

with the error term

$$
E_{t}(k, N)=\left|\mathcal{B}_{t}\right|-\left|\mathcal{B}_{t}^{\prime}\right|
$$

where

$$
\begin{array}{r}
\mathcal{B}_{t}=\{B:\{1, t+1\} \subset B \subset\{1, \ldots, t-1, t+1, \ldots, N-1\},|B|=k-1, \\
t=(t+1)-1 \text { is the only representation of } t \text { in } B-B\}, \\
\mathcal{B}_{t}^{\prime}=\left\{B^{\prime}:\{t, 2 t\} \subset B^{\prime} \subset\{2,3, \ldots, N-1\},\left|B^{\prime}\right|=k-1,\right. \\
\left.\quad t=2 t-t \text { is the only representation of } t \text { in } B^{\prime}-B^{\prime}\right\} .
\end{array}
$$

(2) For $1 \leq t<N / 2$,

$$
E_{t}(k, N)=\left|\mathcal{C}_{t}\right|-\left|\mathcal{C}_{t}^{\prime}\right|
$$

with

$$
\mathcal{C}_{t}=\left\{B \in \mathcal{B}_{t}: 3 t \in B\right\}, \quad \mathcal{C}_{t}^{\prime}=\left\{B^{\prime} \in \mathcal{B}_{t}^{\prime}: 2 t+1 \in B^{\prime}\right\} .
$$

Proof. (1) We divide the sets $B \subset\{0,1, \ldots, N-1\}$ with $|B|=k$ into three classes: 
(i) the sets $B$ such that $0 \notin B$,

(ii) the sets $B$ such that $0 \in B$ and $t \in B$,

(iii) the sets $B$ such that $0 \in B$ and $t \notin B$.

The number of sets in (i) which are counted in $\delta_{t}(k, N)$ is $\delta_{t}(k, N-1)$. The $\left(\begin{array}{c}N-2 \\ k-2\end{array}\right)$ sets in (ii) are all counted in $\delta_{t}(k, N)$. The fact that the sets in (iii) contain 0 is irrelevant because $t$ is not in $B$. So we can cancel 0 , and hence the number of sets in (iii) which are counted in $\delta_{t}(k, N)$ is equal to

$$
|\{B \subset\{1, \ldots, t-1, t+1, \ldots, N-1\}:|B|=k-1, t \in B-B\}| .
$$

Now the sets $B$ in (9) are divided into two classes:

$\left(\mathrm{i}^{\prime}\right)$ the sets $B$ which have a representation $t=b-a$ with $a, b \in B-\{1\}$,

(ii') the sets $B$ which have no such representation, i.e. for which $t=$ $(t+1)-1$ is the only representation of $t$ in $B-B$.

The number of sets in (ii') is $\left|\mathcal{B}_{t}\right|$ by definition. For the description of the number of sets in $\left(i^{\prime}\right)$ we use the map

$$
\begin{gathered}
\phi:\{1, \ldots, t-1, t+1, \ldots, N-1\} \rightarrow\{2,3, \ldots, N-1\}, \\
\phi(1):=t, \quad \phi(x):=x \quad \text { otherwise. }
\end{gathered}
$$

The bijectivity of $\phi$ carries over to the map

$\Phi:\{B \subset\{1, \ldots, t-1, t+1, \ldots, N-1\}:$

$$
\begin{array}{r}
|B|=k-1, \exists a, b \in B-\{1\}: b-a=t\} \\
\rightarrow\left\{B^{\prime} \subset\{2,3, \ldots, N-1\}:\left|B^{\prime}\right|=k-1, \exists a^{\prime}, b^{\prime} \in B^{\prime}-\{t\}: b^{\prime}-a^{\prime}=t\right\}, \\
\Phi(B):=\{\phi(b): b \in B\} .
\end{array}
$$

Hence the number of sets in $\left(\mathrm{i}^{\prime}\right)$ is

$$
\begin{array}{r}
\left|\left\{B^{\prime} \subset\{2,3, \ldots, N-1\}:\left|B^{\prime}\right|=k-1, \exists a^{\prime}, b^{\prime} \in B^{\prime}-\{t\}: b^{\prime}-a^{\prime}=t\right\}\right| \\
=\delta_{t}(k-1, N-2)-\left|\mathcal{B}_{t}^{\prime}\right| \quad \text { by definition of } \mathcal{B}_{t}^{\prime} .
\end{array}
$$

Together we get the recursion formula for $\delta_{t}(k, N)$ with the error term $E_{t}(k, N)=\left|\mathcal{B}_{t}\right|-\left|\mathcal{B}_{t}^{\prime}\right|$.

(2) For $1 \leq t<N / 2$ we use the bijective map

$$
\begin{gathered}
\psi:\{1, \ldots, t-1, t+1, \ldots, N-1\} \rightarrow\{2,3, \ldots, N-1\}, \\
\psi(1):=t, \quad \psi(t+1):=2 t, \quad \psi(2 t):=t+1, \quad \psi(x):=x \quad \text { otherwise }
\end{gathered}
$$

and show:

$$
\Psi: \mathcal{B}_{t}-\mathcal{C}_{t} \rightarrow \mathcal{B}_{t}^{\prime}-\mathcal{C}_{t}^{\prime}, \quad \Psi(B):=\{\psi(b): b \in B\}, \text { is bijective. }
$$

Then part (1) and assertion (10) give at once

$$
\begin{aligned}
E_{t}(k, N) & =\left|\mathcal{B}_{t}\right|-\left|\mathcal{B}_{t}^{\prime}\right|=\left|\mathcal{B}_{t}-\mathcal{C}_{t}\right|+\left|\mathcal{C}_{t}\right|-\left(\left|\mathcal{B}_{t}^{\prime}-\mathcal{C}_{t}^{\prime}\right|+\left|\mathcal{C}_{t}^{\prime}\right|\right) \\
& =\left|\mathcal{C}_{t}\right|-\left|\mathcal{C}_{t}^{\prime}\right|
\end{aligned}
$$


For the proof of (10) we have to show:

(I) $B \in \mathcal{B}_{t}-\mathcal{C}_{t}$ implies $\Psi(B) \in \mathcal{B}_{t}^{\prime}-\mathcal{C}_{t}^{\prime}$,

(II) $B^{\prime} \in \mathcal{B}_{t}^{\prime}-\mathcal{C}_{t}^{\prime}$ implies $\Psi^{-1}\left(B^{\prime}\right) \in \mathcal{B}_{t}-\mathcal{C}_{t}$.

(I) Let $B \in \mathcal{B}_{t}-\mathcal{C}_{t}$. Then $1, t+1 \in B$ and $2 t+1,3 t \notin B$. Application of $\psi$ for $B^{\prime}:=\Psi(B)$ shows that

$$
t, 2 t \in B^{\prime} \text { and } 2 t+1,3 t \notin B^{\prime} .
$$

Hence $t=2 t-t$ is a representation of $t$ in $B^{\prime}-B^{\prime}$, and $B^{\prime} \notin \mathcal{C}_{t}^{\prime}$ because $2 t+1 \notin B^{\prime}$. It remains to show that $t=2 t-t$ is the only representation of $t$ in $B^{\prime}-B^{\prime}$. So let $t=b^{\prime}-a^{\prime}$ be any representation of $t$ with $a^{\prime}, b^{\prime} \in B^{\prime}$. Then

$$
\left\{a^{\prime}, b^{\prime}\right\} \cap\{t, t+1,2 t\} \neq \emptyset .
$$

For otherwise $a^{\prime}$ and $b^{\prime}$ would be invariant under $\psi^{-1}$, and $t=\psi^{-1}\left(b^{\prime}\right)-$ $\psi^{-1}\left(a^{\prime}\right)$ would be a representation of $t$ in $B-B$ which is different from $t=(t+1)-1$. But $a^{\prime} \in\{t+1,2 t\}$ would imply $a^{\prime}+t=b^{\prime} \in B^{\prime}$, and if $b^{\prime} \in\{t, t+1\}$, then $b^{\prime}-t=a^{\prime} \in B^{\prime}$, which both are impossible. Hence by (11), $a^{\prime}=t$ or $b^{\prime}=2 t$, which means $a^{\prime}=t$ and $b^{\prime}=2 t$ because $t=b^{\prime}-a^{\prime}$.

The proof of (II) is exactly the same. Just exchange

$$
t+1 \leftrightarrow 2 t, \quad 2 t+1 \leftrightarrow 3 t, \quad B, \mathcal{B}_{t}, \mathcal{C}_{t}, \psi, \Psi \leftrightarrow B^{\prime}, \mathcal{B}_{t}^{\prime}, \mathcal{C}_{t}^{\prime}, \psi^{-1}, \Psi^{-1}
$$

everywhere and $1 \leftrightarrow t$ at the "right" places, i.e. where $\psi$ is involved.

Remark 1. The sets $\mathcal{B}_{1}$ and $\mathcal{B}_{1}^{\prime}$ in Proposition 2(1) are identical, hence $E_{1}(k, N)=0$, and then the recursion formula yields via induction

$$
\delta_{1}(k, N)=\left(\begin{array}{l}
N \\
k
\end{array}\right)-\left(\begin{array}{c}
N+1-k \\
k
\end{array}\right) .
$$

Similarly one can show

$$
\delta_{2}(k, N)=\left(\begin{array}{l}
N \\
k
\end{array}\right)-\left(\begin{array}{c}
N+1-k \\
k
\end{array}\right)-\left(\begin{array}{c}
N-1-k \\
k-2
\end{array}\right)-\left(\begin{array}{c}
N-3-k \\
k-4
\end{array}\right),
$$

with $\left(\begin{array}{c}m \\ n\end{array}\right):=0$ if $m<0$. Further,

$$
\delta_{M}(k, 2 M)=\left(\begin{array}{c}
2 M \\
k
\end{array}\right)^{*} \text { and } \delta_{M+1}(k, 2 M+1)=\left(\begin{array}{c}
2 M+1 \\
k
\end{array}\right)^{*},
$$

which furnishes a combinatorial interpretation of the coefficients $\left(\begin{array}{l}N \\ k\end{array}\right)^{*}$.

Presumably the sequences $\left(\delta_{t}(k, N)\right)_{0 \leq t<N}$ are almost decreasing. This is easy to show within the interval $N / 2 \leq t<N$, whereas in $0 \leq t \leq N / 2$ there is at least the exception $\delta_{M-1}(M+1,2 M)<\delta_{M}(M+1,2 M), M \geq 3$.

Now we develop a concept to estimate the error terms $E_{t}(k, N)$ of Proposition 2. Let $A$ be a set with $N$ elements. We arrange these elements in a 
scheme

$$
\operatorname{Sch}(A ; N)=\left(a_{i j}\right)_{1 \leq j \leq \ell(i), 1 \leq i \leq t}=\left(\begin{array}{ccccc}
a_{11} & a_{12} & \ldots & \ldots & a_{1, \ell(1)} \\
a_{21} & a_{22} & \ldots & \ldots & a_{2, \ell(2)} \\
\vdots & & & & \\
a_{t 1} & a_{t 2} & \ldots & \ldots & a_{t, \ell(t)}
\end{array}\right)
$$

consisting of $t$ rows of possibly different lengths $\ell(i)$.

In particular, $\operatorname{Sch}(N / t)$ will denote the standard scheme

$$
\operatorname{Sch}(N / t)=\left(\begin{array}{ccccc}
0 & t & 2 t & 3 t & \ldots \\
1 & t+1 & 2 t+1 & 3 t+1 & \ldots \\
2 & t+2 & 2 t+2 & 3 t+2 & \ldots \\
\vdots & & & & \\
t-1 & 2 t-1 & 3 t-1 & 4 t-1 & \ldots
\end{array}\right)
$$

on the set $A=\{0,1, \ldots, N-1\}$.

Schemes $\operatorname{Sch}(A ; N)$ with $r$ rows of length 1 and all rows of length at most 2 will be denoted by $\operatorname{Sch}^{*}(A ; N, r)$. For instance the standard scheme $\operatorname{Sch}(N / t)$ is of type $\operatorname{Sch}^{*}(\{0,1, \ldots, N-1\} ; N, 2 t-N)$ if $(N-1) / 2<t \leq N$.

Two schemes

$\operatorname{Sch}_{1}(A ; N)=\left(a_{i j}\right)_{1 \leq j \leq \ell(i), 1 \leq i \leq t} \quad$ and $\quad \operatorname{Sch}_{2}\left(A^{\prime} ; N\right)=\left(a_{i j}^{\prime}\right)_{1 \leq j \leq \ell^{\prime}(i), 1 \leq i \leq t}$ with the same number $N$ of elements and the same number $t$ of rows are called similar if their rows have the same lengths, i.e. if there exists a permutation $\pi$ on $\{1, \ldots, t\}$ such that $\ell^{\prime}(i)=\ell(\pi(i))$ for $1 \leq i \leq t$.

We call $\operatorname{Sch}_{2}(A ; N)$ finer than $\operatorname{Sch}_{1}(A ; N)$ if $\operatorname{Sch}_{2}(A ; N)$ results from $\operatorname{Sch}_{1}(A ; N)$ by dissection of a row $a_{i 1} \cdots a_{i, \ell(i)}$ of $\operatorname{Sch}_{1}(A ; N)$ into two rows $a_{i 1} \cdots a_{i, m}$ and $a_{i, m+1} \cdots a_{i, \ell(i)}$. Further we require the relation "finer than" to be transitive.

The sets $B \in \mathcal{B}_{t}$ and $B^{\prime} \in \mathcal{B}_{t}^{\prime}$ in Proposition 2 have the following property:

Except 1 and $t+1$ (resp. $t$ and $2 t$ ), $B$ and $B^{\prime}$ do not contain two numbers which are neighbours in any row of the standard scheme $\operatorname{Sch}(N / t)$. Hence generally a subset $B \subset A$ will be called admissible for a given scheme $\operatorname{Sch}(A ; N)$ if and only if $B$ does not contain two elements which are neighbours in any of the rows of $\operatorname{Sch}(A ; N)$.

Our concern will be the cardinality of the sets

$$
\mathcal{P}_{k}(\operatorname{Sch}(A ; N)):=\{B \subset A:|B|=k, B \text { admissible for } \operatorname{Sch}(A ; N)\} .
$$

LEMmA 3. (1) If two schemes $\mathrm{Sch}_{1}$ and $\mathrm{Sch}_{2}$ are similar, then

$$
\left|\mathcal{P}_{k}\left(\operatorname{Sch}_{1}\right)\right|=\left|\mathcal{P}_{k}\left(\operatorname{Sch}_{2}\right)\right|
$$


(2) If the scheme $\mathrm{Sch}_{2}$ is finer than $\mathrm{Sch}_{1}$, then

$$
\mathcal{P}_{k}\left(\mathrm{Sch}_{2}\right) \supset \mathcal{P}_{k}\left(\mathrm{Sch}_{1}\right) .
$$

(3) In particular $r_{2} \geq r_{1}$ implies

$$
\left|\mathcal{P}_{k}\left(\operatorname{Sch}^{*}\left(A ; N, r_{2}\right)\right)\right| \geq\left|\mathcal{P}_{k}\left(\operatorname{Sch}^{*}\left(A^{\prime} ; N, r_{1}\right)\right)\right| .
$$

P r o of. Immediate consequences of the definitions.

Lemma 4. $E_{t}(k, N)=0$ if $t \mid N-1$.

Pr o of. Proposition 2 shows at once $\mathcal{B}_{t}=\mathcal{B}_{t}^{\prime}$ for $t=1$ and $t=N-1$, and $\mathcal{C}_{t}=\mathcal{C}_{t}^{\prime}=\emptyset$ if $N$ is odd and $t=(N-1) / 2$. Thus let $2 \leq t=(N-1) / r \in \mathbb{N}$ and $r \geq 3$. We consider the standard scheme

$\operatorname{Sch}(N / t)$

$$
=\left(\begin{array}{cccccccc}
0 & t & 2 t & 3 t & 4 t & \ldots & (r-1) t & r t \\
1 & t+1 & 2 t+1 & 3 t+1 & 4 t+1 & \ldots & (r-1) t+1 \\
2 & t+2 & 2 t+2 & 3 t+2 & 4 t+2 & \ldots & (r-1) t+2 \\
\vdots & & & & & & \\
t-1 & 2 t-1 & 3 t-1 & 4 t-1 & 5 t-1 & \ldots & r t-1
\end{array}\right)
$$

and apply Proposition 2(2): $\mathcal{C}_{t}$ contains all subsets $B$ of $\{0,1, \ldots, N-1\}-$ $\{0, t\}$ with $|B|=k-1$ and $\{1, t+1,3 t\} \subset B$, which-except 1 and $t+1$-do not contain two neighbouring elements in any of the rows of $\operatorname{Sch}(N / t)$. In particular these sets $B$ do not contain any of the numbers $2 t, 4 t, 2 t+1$. Hence we cancel $0, t, 2 t, 3 t, 1, t+1,2 t+1$ and if possible $4 t$ in $\operatorname{Sch}(N / t)$ and see: $\left|\mathcal{C}_{t}\right|$ counts the sets $B_{0}=B-\{1, t+1,3 t\}$ with $\left|B_{0}\right|=k-4$ which are admissible for the scheme

$\mathrm{Sch}_{1}$

$$
=\left(\begin{array}{cccccccc} 
& & & & & 5 t & \ldots & (r-1) t \\
2 & t+2 & 2 t+2 & 3 t+2 & 4 t+2 & 5 t+2 & \ldots & (r-1) t+2 \\
\vdots & & & & & & & \\
t-1 & 2 t-1 & 3 t-1 & 4 t-1 & 5 t-1 & 6 t-1 & \ldots & r t-1
\end{array}\right) .
$$

Similarly $\mathcal{C}_{t}^{\prime}$ contains all subsets $B^{\prime}$ of $\{0,1, \ldots, N-1\}-\{0,1\}$ with $\left|B^{\prime}\right|=k-1$ and $\{t, 2 t, 2 t+1\} \subset B^{\prime}$, which - except $t$ and $2 t$ - do not contain two neighbouring numbers in any of the rows of $\operatorname{Sch}(N / t)$. In particular, these sets $B^{\prime}$ do not contain $3 t, t+1$, and $3 t+1$. Hence $\left|\mathcal{C}_{t}^{\prime}\right|$ counts the sets $B_{0}^{\prime}=B^{\prime}-\{t, 2 t, 2 t+1\}$ with $\left|B_{0}^{\prime}\right|=k-4$ which are admissible for the scheme 


$$
\operatorname{Sch}_{2}=\left(\begin{array}{cccccccc} 
& & & & 4 t & \ldots & (r-1) t & r t \\
2 & t+2 & 2 t+2 & 3 t+2 & 4 t+2 & \ldots & (r-1) t+2 \\
\vdots & & & & & & \\
t-1 & 2 t-1 & 3 t-1 & 4 t-1 & 5 t-1 & \ldots & r t-1
\end{array}\right) .
$$

The first resp. second row of $\mathrm{Sch}_{1}$ has the same length as the second resp. first row of $\mathrm{Sch}_{2}$. All other rows of $\mathrm{Sch}_{1}$ and $\mathrm{Sch}_{2}$ coincide. Thus $\mathrm{Sch}_{1}$ and $\mathrm{Sch}_{2}$ are similar, and Lemma 3(1) asserts

$$
E_{t}(k, N)=\left|\mathcal{C}_{t}\right|-\left|\mathcal{C}_{t}^{\prime}\right|=\left|\mathcal{P}_{k-4}\left(\mathrm{Sch}_{1}\right)\right|-\left|\mathcal{P}_{k-4}\left(\mathrm{Sch}_{2}\right)\right|=0 .
$$

REMARK 2. A refinement of the argument in the proof of Lemma 4 shows

$$
(-1)^{r} E_{t}(k, N) \geq 0 \quad \text { for } \quad \frac{N-1}{r+1}<t<\frac{N-1}{r}, r=1,2, \ldots
$$

This change of signs in the error terms $E_{t}(k, N)$ makes it difficult to derive an upper bound for $\sum_{t=1}^{N-1} E_{t}(k, N)$ which would be essentially better than the one given in Lemma 7.

LEMMA 5. We have

(1) $E_{t}(k, N)=\left|\mathcal{P}_{k-3}\left(\operatorname{Sch}^{*}(A ; N-4,2 t-N)\right)\right|$ for $(N-1) / 2<t<N-1$.

$$
\sum_{(N-1) / 2<t<N} E_{t}(k, N)=\left(\begin{array}{c}
N-2 \\
k-1
\end{array}\right)^{*}
$$

Proof. (1) For $(N-1) / 2<t<N-1$ Proposition 2(1) shows $\mathcal{B}_{t}^{\prime}=\emptyset$ and hence

$$
E_{t}(k, N)=\left|\mathcal{B}_{t}\right|
$$

Again we consider the standard scheme $\operatorname{Sch}(N / t)$, which is now of type $\operatorname{Sch}^{*}(\{0,1, \ldots, N-1\} ; N, 2 t-N)$.

$\mathcal{B}_{t}$ contains all subsets $B$ of $\{0,1, \ldots, N-1\}-\{0, t\}$ with $|B|=k-1$ and $\{1, t+1\} \subset B$, which - except 1 and $t+1$ - do not contain two numbers in any of the rows of $\operatorname{Sch}(N / t)$. Hence $\left|\mathcal{B}_{t}\right|$ is the number of subsets $B_{0}=$ $B-\{1, t+1\}$ of $A=\{0,1, \ldots, N-1\}-\{0, t, 1, t+1\}$ with $\left|B_{0}\right|=k-3$, admissible for the scheme $\operatorname{Sch}^{*}(A ; N-4,2 t-N)$, which results from $\operatorname{Sch}(N / t)$ by cancellation of the first two rows.

(2) Part (1) shows

$$
E_{t}(k, N)=\sum_{j=0}^{k-3} 2^{j}\left(\begin{array}{c}
N-t-2 \\
j
\end{array}\right)\left(\begin{array}{c}
2 t-N \\
k-3-j
\end{array}\right), \quad(N-1) / 2<t<N-1,
$$

for if $B \in \mathcal{P}_{k-3}\left(\operatorname{Sch}^{*}(A ; N-4,2 t-N)\right)$ contains $j$ elements out of the $N-t-2$ rows of length 2 , for which there are $2^{j}\left(\begin{array}{c}N-t-2 \\ j\end{array}\right)$ possibilities, then 
there are $\left(\begin{array}{c}2 t-N \\ k-3-j\end{array}\right)$ possibilities left for the remaining $k-3-j$ elements of $B$ in the $2 t-N$ rows of length 1 .

Hence with $N=2 M+\delta, 0 \leq \delta \leq 1$, and in view of Lemma 4 ,

$$
\begin{aligned}
\sum_{(N-1) / 2<t<N} E_{t}(k, N) & =\sum_{M+\delta \leq t \leq N-2} \sum_{j \geq 0} 2^{j}\left(\begin{array}{c}
N-t-2 \\
j
\end{array}\right)\left(\begin{array}{c}
2 t-N \\
k-3-j
\end{array}\right) \\
& =\sum_{0 \leq m \leq M-2} \sum_{j \geq 0} 2^{j}\left(\begin{array}{c}
m \\
j
\end{array}\right)\left(\begin{array}{c}
(N-4)-2 m \\
(N-4)-(N-k-1)-j
\end{array}\right) \\
& =\sum_{0 \leq m \leq M-2} \sum_{j \geq 0}(-1)^{j}\left(\begin{array}{c}
m \\
j
\end{array}\right)\left(\begin{array}{c}
N-4-2 j \\
N-k-1
\end{array}\right)
\end{aligned}
$$

(by Lemma 1(2))

$$
\begin{aligned}
& =\sum_{j \geq 0}(-1)^{j}\left(\begin{array}{c}
M-1 \\
j+1
\end{array}\right)\left(\begin{array}{c}
N-2-2(j+1) \\
N-k-1
\end{array}\right) \\
& =\left(\begin{array}{c}
N-2 \\
k-1
\end{array}\right)-\sum_{j \geq 0} 2^{j}\left(\begin{array}{c}
M-1 \\
j
\end{array}\right)\left(\begin{array}{c}
\delta \\
k-1-j
\end{array}\right)
\end{aligned}
$$

(by Lemma 1(2))

$$
=\left(\begin{array}{c}
N-2 \\
k-1
\end{array}\right)^{*}
$$

Lemma 6. We have

$$
\sum_{t=1}^{N-1} E_{t}(k, N) \geq 0 .
$$

Proof. Let $N=2 M+\delta, 0 \leq \delta \leq 1$, and $2 \leq t \leq M-1$. By Proposition 2(1) we have

$$
-E_{t}(k, N) \leq\left|\mathcal{B}_{t}^{\prime}\right|
$$

To estimate $\left|\mathcal{B}_{t}^{\prime}\right|$ we start again by regarding $\operatorname{Sch}(N / t)$. All sets $B \in \mathcal{B}_{t}^{\prime}$ contain $k-1$ numbers, in particular $t$ and $2 t$, and certainly not 0 and 1 . Hence if we cancel $0,1, t, 2 t$ in $\operatorname{Sch}(N / t)$ we obtain a $\operatorname{scheme} \operatorname{Sch}(A ; N-4)$ with at most $t$ rows and such that

$$
\left|\mathcal{B}_{t}^{\prime}\right| \leq\left|\mathcal{P}_{k-3}(\operatorname{Sch}(A ; N-4))\right| .
$$

Now we refine this scheme by cutting every row of length $l \geq 3$ into rows of length 2 and possibly one row of length 1 . The resulting scheme is of type $\operatorname{Sch}^{*}(A ; N-4, \tau(t))$ with some $\tau(t) \leq t$, and Lemma $3(2)$ asserts

$$
\left|\mathcal{P}_{k-3}(\operatorname{Sch}(A ; N-4))\right| \leq\left|\mathcal{P}_{k-3}\left(\operatorname{Sch}^{*}(A ; N-4, \tau(t))\right)\right| .
$$


Therefore, by Lemma 4,

$$
\begin{aligned}
& \sum_{t=1}^{N-1} E_{t}(k, N)= \sum_{2 \leq t \leq M-1} E_{t}(k, N)+\sum_{M+\delta \leq t \leq N-2} E_{t}(k, N) \\
& \geq-\sum_{2 \leq t \leq M-1}\left|\mathcal{P}_{k-3}\left(\operatorname{Sch}^{*}(A ; N-4, \tau(t))\right)\right| \\
&+\sum_{M+1+\delta \leq t \leq N-2}\left|\mathcal{P}_{k-3}\left(\operatorname{Sch}^{*}(A ; N-4,2 t-N)\right)\right| \\
&= \sum_{2 \leq t \leq M-1}\left(\left|\mathcal{P}_{k-3}\left(\operatorname{Sch}^{*}(A ; N-4,2 t-2+\delta)\right)\right|\right. \\
&\left.-\left|\mathcal{P}_{k-3}\left(\operatorname{Sch}^{*}(A ; N-4, \tau(t))\right)\right|\right),
\end{aligned}
$$

and here all summands are nonnegative by Lemma $3(3)$, since

$$
2 t-2+\delta \geq t \geq \tau(t) \quad \text { for } 2 \leq t \leq M-1
$$

LEMMA 7. We have

$$
\sum_{t=1}^{N-1} E_{t}(k, N) \leq\left(\begin{array}{c}
N-1 \\
k-1
\end{array}\right)^{*}
$$

Proof. We already know that

$$
\begin{array}{ll}
E_{t}(k, N)=0 & \text { for } t \mid N-1(\text { by Lemma } 4) \\
E_{t}(k, N) \leq\left|\mathcal{C}_{t}\right| & \text { for } 2 \leq t<(N-1) / 3(\text { by Proposition } 2(2)), \\
E_{t}(k, N) \leq 0 & \text { for } N / 3 \leq t<N / 2 \text { (by Proposition } 2(2))
\end{array}
$$

and

$$
\sum_{(N-1) / 2<t<N} E_{t}(k, N)=\left(\begin{array}{c}
N-2 \\
k-1
\end{array}\right)^{*} \quad(\text { by Lemma } 5(2)) .
$$

Thus all we need is an appropriate upper bound for $\left|\mathcal{C}_{t}\right|, 2 \leq t<$ $(N-1) / 3$. So let us look once more at the standard scheme $\operatorname{Sch}(N / t) . \mathcal{C}_{t}$ contains the subsets $B \subset\{0,1, \ldots, N-1\}-\{0, t\}$ with $|B|=k-1$ and $\{1, t+1,3 t\} \subset B$, which - except 1 and $t+1$ - do not contain two neighbouring numbers in any of the rows of $\operatorname{Sch}(N / t)$. In particular these sets $B$ do not contain the numbers $2 t$ and $2 t+1$. Hence $\left|\mathcal{C}_{t}\right|$ counts certain subsets $B_{0}=B-\{1, t+1,3 t\}$ of $A=\{0,1, \ldots, N-1\}-\{0, t, 1, t+1,3 t, 2 t, 2 t+1\}$ with $\left|B_{0}\right|=k-4$ which are admissible for the scheme $\operatorname{Sch}(A ; N-7)$, resulting from $\operatorname{Sch}(N / t)$ by cancellation of $0, t, 1, t+1,3 t, 2 t$, and $2 t+1$ :

$$
\left|\mathcal{C}_{t}\right| \leq\left|\mathcal{P}_{k-4}(\operatorname{Sch}(A ; N-7))\right| \text {. }
$$

We refine this scheme by cutting every row of length $l \geq 3$ into rows of length 2 and possibly one row of length 1 . Then the resulting scheme is of 
type $\operatorname{Sch}^{*}(A ; N-7, \tau(t))$ with some $\tau(t) \leq t$, for $\operatorname{Sch}(A ; N-7)$ has at most $t$ rows. Then Lemma $3(2)$ asserts

$$
\left|\mathcal{P}_{k-4}(\operatorname{Sch}(A ; N-7))\right| \leq\left|\mathcal{P}_{k-4}\left(\operatorname{Sch}^{*}(A ; N-7, \tau(t))\right)\right| .
$$

Hence for $2 \leq t<(N-1) / 3$,

$$
\left|\mathcal{C}_{t}\right| \leq\left|\mathcal{P}_{k-4}\left(\operatorname{Sch}^{*}(A ; N-7, \tau(t))\right)\right|, \quad \tau(t) \leq t .
$$

On the other hand, Lemma 5 with $N-3$ and $k-1$ instead of $N$ and $k$ yields

$$
\begin{aligned}
\left(\begin{array}{c}
N-5 \\
k-2
\end{array}\right)^{*} & =\sum_{(N-4) / 2<t<N-4}\left|\mathcal{P}_{k-4}\left(\operatorname{Sch}^{*}(A ; N-7,2 t-N+3)\right)\right| \\
& =\sum_{2-\delta \leq t \leq M-2}\left|\mathcal{P}_{k-4}\left(\operatorname{Sch}^{*}(A ; N-7,2 t+\delta-3)\right)\right|
\end{aligned}
$$

(by substitution $t \mapsto t+M+\delta-3$ with $N=2 M+\delta, 0 \leq \delta \leq 1$ )

$$
\geq \sum_{2 \leq t<(N-1) / 3}\left|\mathcal{P}_{k-4}\left(\operatorname{Sch}^{*}(A ; N-7,2 t+\delta-3)\right)\right| .
$$

Therefore by (15) and (16),

$$
\begin{aligned}
\left(\begin{array}{c}
N-5 \\
k-2
\end{array}\right)^{*}- & \sum_{2 \leq t<(N-1) / 3}\left|\mathcal{C}_{t}\right| \\
& \geq \sum_{2 \leq t<(N-1) / 3}\left(\left|\mathcal{P}_{k-4}\left(\operatorname{Sch}^{*}(A ; N-7,2 t+\delta-3)\right)\right|\right. \\
& \left.-\left|\mathcal{P}_{k-4}\left(\operatorname{Sch}^{*}(A ; N-7, \tau(t))\right)\right|\right),
\end{aligned}
$$

and by Lemma 3(3), all summands here are nonnegative, since $t \geq \tau(t)$ and thus $2 t+\delta-3 \geq \tau(t)$. This is obvious for $t \geq 3$ and also for $t=2$ and $\delta=1$. But if $t=2$ and $\delta=0$, then $N-7$ is odd and hence $\tau(2)=1$.

This shows

$$
\sum_{2 \leq t<(N-1) / 3}\left|\mathcal{C}_{t}\right| \leq\left(\begin{array}{c}
N-5 \\
k-2
\end{array}\right)^{*}
$$

and combined with the estimates at the beginning of the proof and with Lemma 2(2) we finally get

$$
\sum_{t=1}^{N-1} E_{t}(k, N) \leq\left(\begin{array}{c}
N-2 \\
k-1
\end{array}\right)^{*}+\left(\begin{array}{c}
N-5 \\
k-2
\end{array}\right)^{*} \leq\left(\begin{array}{c}
N-1 \\
k-1
\end{array}\right)^{*}
$$

Proposition 3. (1) For $2 \leq k \leq N$ and suitable $\theta \in[0,1]$, $D(k, N)=D(k, N-1)+D(k-1, N-2)+(2 N-1)\left(\begin{array}{c}N-2 \\ k-2\end{array}\right)+2 \theta\left(\begin{array}{c}N-1 \\ k-1\end{array}\right)^{*}$. 
(2) Explicitly for $1 \leq k \leq N$ and $\theta \in[0,1]$,

$$
D(k, N)=D_{0}(k, N)+2 \theta\left(\begin{array}{c}
N+1 \\
k+1
\end{array}\right)^{*}
$$

with

$$
D_{0}(k, N)=(2 N+1)\left(\begin{array}{l}
N \\
k
\end{array}\right)-2\left(\begin{array}{c}
N \\
k+1
\end{array}\right)-2\left(\begin{array}{c}
N+2 \\
k+2
\end{array}\right)+2\left(\begin{array}{c}
N+2-k \\
k+2
\end{array}\right) .
$$

Proof. (1) Clearly

$$
D(k, N)=\left(\begin{array}{l}
N \\
k
\end{array}\right)+2 \sum_{k=1}^{N-1} \delta_{t}(k, N),
$$

since $D(k, N)=\sum_{-N+1 \leq t \leq N-1} \delta_{t}(k, N)$ by definition and

$$
\delta_{0}(k, N)=\left(\begin{array}{c}
N \\
k
\end{array}\right), \quad \delta_{-t}(k, N)=\delta_{t}(k, N) .
$$

Therefore the recursion formula in Proposition 2(1) gives

$$
\begin{aligned}
& D(k, N)=\left(\begin{array}{l}
N \\
k
\end{array}\right)+2 \sum_{t=1}^{N-1}\left(\delta_{t}(k, N-1)+\delta_{t}(k-1, N-2)\right) \\
&+2(N-1)\left(\begin{array}{c}
N-2 \\
k-2
\end{array}\right)+2 \sum_{t=1}^{N-1} E_{t}(k, N) \\
&= 2 \sum_{t \geq 1} \delta_{t}(k, N-1)+2 \sum_{t \geq 1} \delta_{t}(k-1, N-2) \\
&+(2 N-2)\left(\begin{array}{c}
N-2 \\
k-2
\end{array}\right)+\left(\begin{array}{c}
N-1 \\
k
\end{array}\right) \\
&+\left(\begin{array}{c}
N-2 \\
k-1
\end{array}\right)+\left(\begin{array}{c}
N-2 \\
k-2
\end{array}\right)+2 \theta\left(\begin{array}{c}
N-1 \\
k-1
\end{array}\right) * \\
&(\text { with } \theta \in[0,1], \text { by Lemmata } 6 \text { and } 7) \\
& D(k, N-1)+D(k-1, N-2) \\
&+(2 N-1)\left(\begin{array}{c}
N-2 \\
k-2
\end{array}\right)+2 \theta\left(\begin{array}{c}
N-1 \\
k-1
\end{array}\right)^{*} \quad \text { (by (17)). }
\end{aligned}
$$

(2) The initial values are

$$
D(1, N)=N=D_{0}(1, N), \quad N \geq 1,
$$

and

$$
D(k, k)=2 k-1=D_{0}(k, k), \quad k \geq 1 .
$$

The rest is straightforward induction on $k$ and $N$ with the use of the recursion formula of part (1) and Lemma 2(2) for the $\theta$-terms. 
Now we use the recursion formulae of $S(k, N)$ and $D(k, N)$ to prove:

THEOREM. We have

$$
1 \leq \frac{D(k, N)}{S(k, N)}<2 \quad \text { for } 1 \leq k \leq N .
$$

Pr o of. First we show

$$
\Delta_{1}(k, N):=D(k, N)-S(k, N) \geq 0 \quad \text { for } 1 \leq k \leq N
$$

by induction on $k$ and $N$. The initial values are

$$
\begin{aligned}
\Delta_{1}(1, N) & =D(1, N)-S(1, N)=N-N=0, \\
\Delta_{1}(k, k) & =D(k, k)-S(k, k)=(2 k-1)-(2 k-1)=0,
\end{aligned}
$$

and the induction step $N-1 \mapsto N$ with $N=2 M+\delta>k \geq 2$ is $\Delta_{1}(k, N)=D(k, N)-S(k, N)$

$$
\begin{aligned}
\geq & D(k, N-1)+D(k-1, N-2)+(2 N-1)\left(\begin{array}{c}
N-2 \\
k-2
\end{array}\right) \\
& -S(k, N-1)-S(k-1, N-2) \\
& -(2 N-1)\left(\begin{array}{c}
N-2 \\
k-2
\end{array}\right)-2^{k-1}\left(\begin{array}{c}
M-1 \\
k-1
\end{array}\right)+2\left(\begin{array}{c}
N-2 \\
k
\end{array}\right)^{*}
\end{aligned}
$$

(by Proposition 3(1) and Proposition 1(2))

$$
\begin{aligned}
& =\Delta_{1}(k, N-1)+\Delta_{1}(k-1, N-2)-2^{k-1}\left(\begin{array}{c}
M-1 \\
k-1
\end{array}\right)+2\left(\begin{array}{c}
N-2 \\
k
\end{array}\right)^{*} \\
& \geq 2\left(\begin{array}{c}
2 M-2 \\
k
\end{array}\right)^{*}-2^{k-1}\left(\begin{array}{c}
M-1 \\
k-1
\end{array}\right)
\end{aligned}
$$

(by induction hypothesis and Lemma 2(2))

$$
\begin{aligned}
& \geq 2^{k-1}(k-2)\left(\begin{array}{c}
M-1 \\
k-1
\end{array}\right) \quad(\text { by Lemma } 2(5)) \\
& \geq 0 .
\end{aligned}
$$

Finally we prove

$$
\Delta_{2}(k, N):=2 S(k, N)-D(k, N)>0 \quad \text { for } 1 \leq k \leq N,
$$

again by induction on $k$ and $N$, and by Propositions 1(2) and 3(1):

$$
\begin{aligned}
\Delta_{2}(1, N) & =2 N-N=N>0, \\
\Delta_{2}(k, k) & =2(2 k-1)-(2 k-1)=2 k-1>0,
\end{aligned}
$$

and the induction step $N-1 \mapsto N$ with $N=2 M+\delta>k \geq 2$ is

$$
\begin{aligned}
\Delta_{2}(k, N) & =2 S(k, N)-D(k, N) \\
& \geq 2 S(k, N-1)+2 S(k-1, N-2)+2(2 N-1)\left(\begin{array}{c}
N-2 \\
k-2
\end{array}\right)
\end{aligned}
$$




$$
\begin{aligned}
& +2^{k}\left(\begin{array}{c}
M-1 \\
k-1
\end{array}\right)-4\left(\begin{array}{c}
N-2 \\
k
\end{array}\right) * \\
& -D(k, N-1)-D(k-1, N-2) \\
& \quad-(2 N-1)\left(\begin{array}{c}
N-2 \\
k-2
\end{array}\right)-2\left(\begin{array}{c}
N-1 \\
k-1
\end{array}\right) \\
& =\Delta_{2}(k, N-1)+\Delta_{2}(k-1, N-2)+(2 N-1)\left(\begin{array}{c}
N-2 \\
k-2
\end{array}\right) \\
& \quad+2^{k}\left(\begin{array}{c}
M-1 \\
k-1
\end{array}\right)-4\left(\begin{array}{c}
N-2 \\
k
\end{array}\right)^{*}-2\left(\begin{array}{c}
N-1 \\
k-1
\end{array}\right)^{*} \\
& >0
\end{aligned}
$$

by induction hypothesis and Lemma $2(6)$.

REMARK 3. The development of $S(k, N)$ in Proposition 1(1) and of $D_{0}(k, N)$ in Proposition 3(2) in powers of $N$ yields, as $N \rightarrow \infty$,

$$
\begin{aligned}
S(k, N) & =\left(\begin{array}{c}
k+1 \\
2
\end{array}\right)\left(\begin{array}{l}
N \\
k
\end{array}\right)+O\left(N^{k-1}\right), \\
D_{0}(k, N) & =\left(k^{2}-k+1\right)\left(\begin{array}{c}
N \\
k
\end{array}\right)+O\left(N^{k-1}\right) .
\end{aligned}
$$

The appearance of the coefficients $\left(\begin{array}{c}k+1 \\ 2\end{array}\right)$ and $k^{2}-k+1$ is not surprising: If $N$ is large compared to $k$, then within most of the $\left(\begin{array}{l}N \\ k\end{array}\right)$ subsets $A$ of $\{0,1, \ldots, N-1\}$ with $|A|=k$ there are only very few nontrivial coincidences $a+b=a^{\prime}+b^{\prime}$. In particular sets with $|A|=k$ and without such coincidences have $S(A)=\left(\begin{array}{c}k+1 \\ 2\end{array}\right)$ and $D(A)=k^{2}-k+1$ (comp. introduction). Therefore the upper bound $D(k, N) \leq\left(k^{2}-k+1\right)\left(\begin{array}{l}N \\ k\end{array}\right)$ is obvious. On the other hand, $D(k, N) \geq D_{0}(k, N)$ by Proposition $3(2)$, which together with (19) yields

$$
D(k, N)=\left(k^{2}-k+1\right)\left(\begin{array}{l}
N \\
k
\end{array}\right)+O\left(N^{k-1}\right) .
$$

This and (18) imply at once

$$
\lim _{N \rightarrow \infty} \frac{D(k, N)}{S(k, N)}=1+\left(1-\frac{2}{k}\right)\left(1-\frac{2}{k+1}\right)
$$

and

$$
\lim _{k \rightarrow \infty} \lim _{N \rightarrow \infty} \frac{D(k, N)}{S(k, N)}=2 .
$$

On the other hand, the explicit formulae for $S(k, N)$ and $D(k, N)$ show immediately that there exists a positive constant $c_{0}$ such that for all $N \geq 1$,

$$
1 \leq \frac{D(k, N)}{S(k, N)}<1+\frac{c_{0}}{N} \quad \text { for } N / 2<k \leq N
$$


Hence the lower bound 1 as well as the upper bound 2 of the Theorem are best possible, and their values are not caused by accidental irregularities of the quotient $D(k, N) / S(k, N)$ for small values of $k$ and $N$.

\section{References}

[1] G. A. Freı̆man and V. P. Pigarev, The relation between the invariants $R$ and T, in: Number-Theoretic Studies in the Markov Spectrum and in the Structural Theory of Set Addition, Kalinin. Gos. Univ., Moscow, 1973, 172-174 (in Russian).

[2] R. R. Hall and G. Tenenbaum, Divisors, Cambridge Univ. Press, 1988.

[3] E. Landau, Handbuch der Lehre von der Verteilung der Primzahlen, 2. Band, Teubner, Leipzig, 1909.

[4] J. Riordan, Combinatorial Identities, Krieger, Huntington, 1979.

[5] I. Z. Ruzsa, Sets of sums and differences, in: Séminaire de Théorie des Nombres de Paris, 1982-83, Birkhäuser, Boston, 1984, 267-273.

Zentrum Mathematik

Technische Universität München

D-80290 München, Germany

E-mail: roeslerf@mathematik.tu-muenchen 